inflammatory polyarthritis, however, remains unclear. So, Franklin et al. measured lymphoma risk in an unselected cohort of patients with inflammatory polyarthritis, and evaluated the effects of disease severity and treatment history on this risk.

This prospective, primary-care-based study recruited 2,105 patients enrolled on the Norfolk Arthritis Register (NOAR) between 1990 and 1999, who were recently diagnosed with inflammatory polyarthritis, and had accessible hospital records. During annual follow-up, data on prescription drug use and disease severity were collected.

After a total follow-up of 15,548 personyears, the incidence of lymphoma was 7.07 cases per 10,000 person-years. Lymphoma risk was highest in patients who had ever tested positive for rheumatoid factor, those ever diagnosed with RA, or those who had ever received disease-modifying antirheumatic drugs. Methotrexate use carried the highest risk ( 5 times that of the general population), although all these factors were interrelated.

The small number of lymphoma cases, however, meant that the authors could not draw definitive conclusions about the effects of disease severity and drug exposure on lymphoma risk. They highlight the need for appropriate control groups in future studies that explore the effects of antirheumatic drugs on lymphoma risk.

Original article Franklin J et al. (2006) Incidence of lymphoma in a large primary care derived cohort of cases of inflammatory polyarthritis. Ann Rheum Dis 65: 617-622

\section{IL4R mutations predict erosive disease in rheumatoid arthritis}

Early treatment of inflammation in patients with rheumatoid arthritis (RA) is thought to limit the progression of joint destruction, so prompt identification of patients at risk of developing erosive disease is important. Genome-wide studies have suggested that the interleukin 4 receptor (IL4R) gene is associated with susceptibility to and/or progression of disease in RA. Prots et al. investigated the influence of two known single nucleotide polymorphisms in IL4R (the resulting amino-acid substitutions Ile50Val and Gln551Arg cause functional changes in its product, IL-4R) on RA susceptibility and progression.
This multicenter study enrolled 471 patients with RA and 371 controls. The presence of erosive disease was determined by examination of hand and foot radiographs taken 2 years after disease onset.

Neither mutation was associated with RA susceptibility, but the Ile50Val variant of IL-4R was associated with the development of erosive disease within the first 2 years of the disease (odds ratio 3.86). Two copies of the allele that resulted in the Ile50Val substitution conferred more than twice the risk observed with one copy; the predictive value of a single copy of this allele for the development of early erosive changes in RA was similar to that of existing markers (including rheumatoid factor).

The authors found that the lle50Val mutation impaired the function of IL-4R, reducing its response to IL-4. They suggest that this impairment could cause an increase in $T_{H^{1-}}$ mediated effects of IL-4 (at the expense of $\mathrm{T}_{\mathrm{H}^{2}}{ }^{2}$ mediated effects), which could, in turn, worsen inflammation and cause rapid joint damage.

Original article Prots I et al. (2006) Association of the IL4R single-nucleotide polymorphism I50V with rapidly erosive rheumatoid arthritis. Arthritis Rheum 54: 1491-1500

\section{The course of juvenile idiopathic arthritis can be predicted soon after diagnosis}

A single-center study by Singh-Grewal and colleagues has confirmed the existence of monophasic, polycyclic, and persistent disease courses in systemic juvenile idiopathic arthritis (JIA). The team also found that early features of JIA can predict both disease course and the time to remission.

They recruited 45 children (19 male) diagnosed with systemic JIA between 1996 and 2000. Mean follow-up was 4.88 years (data from two children who died within 2 years of the study start were included).

The authors retrospectively compared several remission criteria, and found that inactive disease while not receiving medication for 3 months was the most useful definition for determining the disease course of patients with JIA. These remission criteria identified patients with monophasic JIA with $89.7 \%$ specificity and $100 \%$ sensitivity, despite only requiring disease to be inactive for 3 months. The authors used 